\title{
¿Sobre qué bases mínimas podría construirse un acuerdo global cosmopolita?
}

\section{(On What Minimum Bases Could a Cosmopolitan Global Agreement Be Built?)}

\author{
Juan Antonio FernándeZ MANZANO
}

Recibido: 11 de enero de 2013

Aceptado: 4 de abril de 2013

\section{Resumen}

El artículo explora algunos de los principales rasgos políticos de la época globalizada y defiende la necesidad de entidades políticas representativas que superen el marco del Estado nación. A continuación se pregunta bajo qué presupuestos ético-políticos podría defenderse un modelo político-jurídico global para un mundo axiológicamente diverso y qué concepciones políticas y valores podrían proponerse como referente normativo para una gobernanza global cuya meta habría de ser el ejercicio legítimo del poder. Teniendo en cuenta las dificultades de tal empresa, se presentan posibles estrategias y razones que invitan a considerar que la posibilidad de un acuerdo global no es del todo implausible.

Palabras clave: Globalización, Estado global, cosmopolitismo, valores mínimos, pluralismo.

\begin{abstract}
This article explores some of the main political features of the globalized era and defends the need for representative political institutions that exceed the nation state. The following question is under what ethico-political conditions we could defend a political and legal framework for an axiologically diverse world and what political conceptions and values could be proposed as a normative model for global governance whose goal ought to be the legitimate exercise of power.
\end{abstract}


Considering the difficulties of such an undertaking, possible strategies and reasons are presented in order to contemplate that the possibility of a global agreement is not entirely implausible.

Keywords: Globalization, Global State, cosmopolitanism, minimum values, pluralism.

El presente trabajo estudia en primer lugar las razones que respaldan la necesidad de un ordenamiento jurídico político internacional para a continuación ocuparse de delinear las bases normativas sobre las que podría edificarse.

Procediendo por la vía negativa, comenzamos defendiendo que el modelo estatal vigente es un marco insuficiente en el actual contexto global. Las sociedades limitadas y organizadas dentro de las fronteras de sus respectivos Estados, propias de la primera modernidad, se han convertido en sociedades insertadas y mezcladas, velis nolis, con otras sociedades y formas de vida transnacionales. Las fronteras son porosas al dinero, las tecnologías y la información y por su parte, las empresas y bancos operan más allá de los territorios en los que fueron creados. Los procesos de globalización clausuran por consiguiente el modelo estatal westafliano con sus esquemas definidos y clausurados. ${ }^{1}$

Esto es constatable desde cuatro perspectivas diferentes. En primer lugar, los Estados nacionales no pueden desmarcarse de la influencia de los heterogéneos procesos transnacionales. La línea que habitualmente se trazaba entre los asuntos propios y los ajenos aparece desdibujada; lo que acontece en cualquier parte del mundo a menudo tiene ecos en otros lugares. ${ }^{2}$ La actual crisis económica mundial ha puesto de manifiesto que un fallo en una parte del sistema tiene efectos contundentes en todas las demás.

Este no es el único caso: muchas otras decisiones "locales" como la tala de zonas arboladas, la construcción de centrales nucleares o la modificación de los tipos de interés, tienen repercusiones internacionales que ponen en entredicho la tradicional incomunicabilidad de los Estados. ${ }^{3}$ Por lo que se refiere a la capacidad de afectarse mutuamente, el actual es un contexto carente de "afueras".

En segundo lugar, la insuficiencia del modelo de estados independientes se hace también patente ante la existencia de cuestiones que sobrepasan con mucho la capacidad de los Estados. Nos estamos refiriendo a problemas como las epidemias, las cada vez más frecuentes catástrofes medioambientales, las bolsas de refugiados, las políticas de seguridad, los conflictos bélicos, las mafias, los delitos a través de inter-

\footnotetext{
1 Toulmin (2001, p. 288).

2 Cf. Bauman (1999, 2009).

3 Cf. Held (2007, p. 409).
} 
net, la amenaza terrorista, los paraísos fiscales, etc. Se trata de retos heterogéneos que inauguran un escenario global unificado al que Beck llama "la sociedad del riesgo mundial"4 y Giddens califica como "el precipicio de la historia". 5 Al margen de lo apocalíptico de tales enunciaciones, lo que nos interesa destacar es que pretender hallar soluciones estatales a los desafíos globales es errar en la escala con la que estos pueden abordarse. Por su trascendencia y magnitud, estos asuntos no pueden recibir tratamientos unilaterales; su propia existencia demanda una institución global con un acercamiento comprehensivo y no parcial.

En tercer lugar, la instancia marco desde donde tradicionalmente se realizaba la gestión de lo común, el Estado-nación, ha sufrido un progresivo y notable cambio de carácter. Si en sus inicios la nota fundamental del Estado era su soberanía, esto es, su capacidad para no reconocer ninguna instancia superior interna o externamente, en la "segunda modernidad" tal poder ha dejado de ser, como demandaba Bodin, perpetuo y absoluto. 6 Las atribuciones clásicas del Estado moderno: moneda, política exterior, justicia y ejército han dejado de ser competencia interna y se han delegado, como sucede en el caso de los países de la Unión Europea, a instancias supranacionales. Con los recientes flujos de poder, especialmente desde los años noventa, los Estados nacionales han cedido áreas competenciales que anteriormente les estaban reservadas en exclusividad. Como resultado de ello, han aparecido nuevos centros de decisión regional, mas congruentes con la magnitud de los problemas a abordar. Aunque pudiera parecer una paradoja, para poder encarar y resolver muchos de los problemas actuales, los Estados se han visto forzados a traspasar áreas de soberanía a instancias políticas superiores para recuperarlas en el concierto internacional. Beck se refiere a esta estrategia como de autoafirmación por medio de la auto-desposesión. ${ }^{7}$ En efecto, los Estados reconfiguran sus atribuciones habituales y buscan formas concertadas de actuación porque es un medio efectivo para ampliar su capacidad de influencia sobre asuntos que de otro modo escaparían a su alcance. 8

Más delicado es, en cuarto lugar, cómo la autoridad soberana de los Estados se ve comprometida desde otros flancos, puesto que no se trata ya de una transferen-

\footnotetext{
${ }^{4}$ La civilización se pone, afirma Beck, en peligro a sí misma y las amenazas se ciernen sobre todos. Beck (1998, p. 65) y Beck (2008). Una afirmación que, no obstante, conviene matizar puesto que, aunque estos factores influyen universalmente, no lo hacen en la misma medida: los países más desarrollados por lo general están más protegidos, al menos en el corto plazo.

5 Giddens se refiriere a que por primera vez en el medio millón de años de la historia de la humanidad se ha llegado a la posibilidad de que esta pueda desaparecer abruptamente Giddens (1987, p. 182).

6 Bodin (1985).

7 Cf. Beck (1998, p. 188).

8 En asuntos como la evasión de impuestos, la delincuencia económica o la lucha contra el paro, por citar algunos ejemplos.
} 
cia de soberanía para obtener algo a cambio, sino que cabría hablar más bien de una pérdida involuntaria de atribuciones. Nos referimos a la magra potestad que retienen los Estados en lo referido a intereses tan sensibles como los factores primarios de la producción y el intercambio. Capital, tecnología, personas y bienes se mueven con facilidad a través de los límites nacionales y el Estado-nación posee cada vez menos autoridad para regular sus flujos. ${ }^{9}$

Discrepamos con la idea de que las funciones del Estado y sus mecanismos regulatorios hayan continuado dirigiendo el reino de la producción económica y social y del intercambio. Ni siquiera los Estados-nación más poderosos pueden ser considerados como autoridades supremas y soberanas dentro o fuera de sus fronteras y tampoco parece que los organismos nacionales y supranacionales estén unidos bajo una única lógica de mando. ${ }^{10}$ Por el contrario, los hechos apuntan a la falta de control general de los procesos a escala global. Lo que habría sería más parecido a una malla de relaciones yuxtapuestas de poder, sin mando único, con un disfuncional solapamiento de competencias que es fuente de continuas tensiones. ${ }^{11}$ Antes que de un imperio, podría hablarse de un sistema multicéntrico de "estructura reticular" no tan lejano a la fragmentación de poder de las poliarquías medievales. La analogía es pertinente porque tanto entonces como ahora, lo que destaca es la ausencia de una estructura de poder única y soberana. 12

Ahora bien, a diferencia de lo que sucedía la época medieval, el grado de interdependencia global actual es mucho mayor y en consecuencia, mayor es también el número de posibles arbitrariedades. El modo actual en el que los Estados operan, con acuerdos coyunturales y parciales, y sin un sistema de derecho común está cada vez más cerca de un modelo anárquico o de estado de naturaleza que de una sociedad política plenamente constituida. ${ }^{13}$ El reparto de poder, al no contar con instituciones político-jurídicas globalmente legitimadas y con influencia global, retroalimenta el surgimiento de focos de poder privados y auto-interesados que operan al margen del control político. Al dejar los Estados de ser los polos en los que se concentra el binomio política-poder, ${ }^{14}$ las fuerzas extra-estatales, cuya relevancia siempre será directamente proporcional al poder que absorban de los Estados, aprovechan para operar libremente.

\footnotetext{
9 Sassen (2007, pp. 326 ss).

10 Tal sería la tesis de Hardt y Negri (2002).

11 El número de organizaciones gubernamentales y no gubernamentales internacionales ha aumentado exponencialmente desde comienzos del siglo XX. Cf. Held (2007, p. 418).

$12 C f$. Held (2005, p. 107). En parecidos términos encontramos el texto de del Águila y Vallespín (2006, p. 533).

13 Así lo cree Giddens (1987, p. 180): "We live not only in a world capitalist economy but in a world politico-military order of anarchically organized nation-states".

14 Biehl (1998, p. 117).
} 
La ausencia de entidad política arbitral apoyada en bases democráticas con suficiente reconocimiento, autonomía y capacidad para hacer frente a los retos globales y para ejercer como poder arbitral es un vacío de poder que deja en situación de indefensión a los más débiles, al no poder ni valerse por sus propios medios ni estar en posición de exigir responsabilidades a nadie, lo que en consecuencia separa cada vez más a las elites mundiales sin fronteras de los perdedores. ${ }^{15}$

A la luz de lo expuesto podemos concluir afirmado que un mundo con un deficiente o incompleto ordenamiento político-jurídico global sería menos injusto que un mundo sin él.

Si se aceptan las premisas anteriores, que dibujan un contexto general que podríamos resumir como "interdependencia desregulada", la cuestión siguiente sería la de elucidar bajo qué presupuestos éticos y políticos podría defenderse la integración política y la creación de un marco constitucional global.

Si entendemos que dicho marco habría de ocuparse al menos de articular los derechos y obligaciones que unos individuos tienen con otros, lo primero que deberíamos hacer es abordar la cuestión preliminar relacionada con los límites de la responsabilidad mutua entre individuos, esto es, ¿hasta dónde debe llegar, en caso de aceptarse, la responsabilidad de cada individuo hacia el resto de seres humanos, con independencia de su procedencia, cercanía u otros factores similares?

Desde Diógenes, las propuestas cosmopolitas parten de afirmar que no es moralmente aceptable la indiferencia absoluta hacia los problemas de los desconocidos. Para defender esta consideración nos parece oportuno recordar cómo la vulnerabilidad esencial del hombre y su situación estructuralmente relacional son dos condiciones inseparables sin las que no podría entenderse el desarrollo de las facultades morales. Los individuos no son autosuficientes y requieren de la ayuda de otros. Este hecho les coloca en posición de dar cuenta ante los demás de sus actos y de aceptar que los afectados por ellos demanden explicaciones. El concepto de responsabilidad va ligado a la apertura de un diálogo en el que se piden y ofrecen explicaciones. La creciente interdependencia a la que aludíamos unas líneas atrás no hace sino ampliar el conjunto de los afectados y nos lleva a defender la idea de que el mundo es algo más que un colectivo de seres humanos desvinculados entre sí y divididos por Estados, ideologías o religiones. La noción estoica de koimos nomos o derecho común para la humanidad, el ius gentium romano o el ius cosmopoliticum kantiano ya apuntaban a la idea de que es necesario considerar a los individuos como entes interconectados con capacidad para diseñar escenarios políticos justos en los que sea posible cooperar y auxiliarse mutuamente.

Dos de los requisitos formales de la idea de justicia: imparcialidad e inclusión instan a considerar lo humano en su conjunto conformando una metaunidad en cuya

15 Höffe (2007, p. 283). 
base se encontrarían individuos igualmente valiosos con independencia de su lugar de origen u otras consideraciones idiosincráticas. Estos requisitos formales de la justica están apoyados por los sentimientos espontáneos de simpatía y defensa mutua. Indudablemente, la moralidad de tales sentimientos no estriba en su mera ocurrencia con los seres cercanos, sino en su extensión universal. Los sentimientos naturales se moralizan cuando se hacen inclusivos y tienen en cuenta a todos los demás. ${ }^{16}$ En ese sentido, la no indiferencia nos parece un punto de partida aceptable.

Partiendo de esta posición de partida, existen propuestas, como la que Peter Singer presenta en su trabajo "Famine, Affluence and Morality"17 y que Peter Unger desarrolla en su Living High and Letting Die: Our Illusion of Innocence, 18 según las cuales, la no indiferencia se traduce en un compromiso muy alto con los demás; hasta el punto de formular como imperativo hacer todo lo posible para aliviar la situación de quienes están en peores circunstancias. Siguiendo este argumento ético, sería incorrecto que alguien empleara sus recursos en actividades no primarias para dedicarlo a aliviar la situación de quienes sufren penalidades en cualquier lugar del mundo. Lo que en un caso particular podría resultar aceptable, se vuelve excesivamente pesado si se eleva a principio normativo universal. Llevados a este extremo, podemos imaginar que esta regla exige a los individuos deshacerse de prácticamente todos sus bienes e incluso dedicar la mayor parte de su tiempo con el fin de evitar males mayores a otros.

Creemos que la no indiferencia no tiene que significar que la intensidad de la solidaridad hacia los desconocidos haya de ser la misma que despiertan los allegados, por quienes generalmente alguien está dispuesto a hacer mayores sacrificios. 19 Por tanto, un ordenamiento político o jurídico global, para no ser una utopía irrealizable y tener posibilidades de ejecutarse, no puede estar fundado en un rigorismo moral semejante. No es razonable pretender universalizar un grado de afección similar al que alguien siente, y tiene derecho a sentir, por sus seres más cercanos.

Por tanto, las respuestas a la pregunta inicial acerca del grado de responsabilidad exigible hacia los extraños debe buscar, para poder ser implementada, una solución prudencial que ocupe un término medio entre la responsabilidad de todos ante todo y el absoluto desentendimiento de quienes, como Nozick, defienden "the fact of our separate existences". 20

Una formulación menos exigente, y más fácilmente aceptable como punto de partida podría expresarse del siguiente modo: la obligación moral recae sobre quien

\footnotetext{
$16 \operatorname{Mill}(1997$, pp. 114 ss).

17 Singer (1972).

18 Unger (1996).

19 Cf. Appiah (2007, p. 206).

20 Nozick (1999, p. 33).
} 
está en posición de evitar algo horrible a un bajo coste. ${ }^{21}$ No es moralmente aceptable omitir el auxilio a quien está en una situación desesperada cuando puede hacerse algo sin mucho esfuerzo. Mill lo calificaba la omisión de socorro como "selfish abstinence".22

Nos parece que esta postura puede ser defendida con más facilidad puesto que no demanda conductas heroicas, sino razonablemente comprometidas.

Si buscamos la traslación de esta idea en el plano político, podríamos plasmarla en el establecimiento global de una suerte de deberes samaritanos, cuyas exigencias podrían ser generalmente compartibles. Tales deberes permitirían emprender acciones colectivas que evitaran crímenes, catástrofes humanas o violaciones de derechos humanos si el sacrificio que demandaran no fuera excesivamente exigente para quien lo prestara. ${ }^{23}$ Este principio básicamente reclamaría comprometerse con la asistencia a otros en determinadas circunstancias: "... when they are in dire need and one can help them at no unreasonable cost to oneself'.24 Para ser más precisos, podemos distinguir cuatro condiciones: en primer lugar, que el sujeto, individual o colectivo, se vea expuesto a un peligro de proporciones significativas que comprometa la satisfacción mínima de ciertas preferencias básicas. En segundo lugar, el sujeto necesitado de ayuda no debe hallarse en tales circunstancias por culpa suya, de manera intencional o por negligencia. En tercer lugar, que la ayuda que requiere sea la estrictamente necesaria para superar el peligro y no esté a su alcance y por último, en cuarto lugar, que los costes de prestar tal ayuda no coloquen a quien auxilia en una situación comprometida. La conjunción de las cuatro puede sintetizarse en la siguiente formulación: adquieren derechos samaritanos quienes se enfrentan a una situación de peligro de la que no son responsables y cuando los únicos medios de superarla implican la ayuda que otros pueden ofrecer a un coste razonable. 25

Los derechos samaritanos que defendemos dan una respuesta más satisfactoria a la creencia en que cada ser humano tiene algún tipo de responsabilidad moral para con el resto de congéneres, alejada tanto de las exigencias leoninas de Unger como del desentendimiento mutuo.

Aceptando la pertinencia del marco político-jurídico global y la exigencia moral de establecer obligaciones mutuas a través de normas universales de convivencia, nos topamos con el problema de cómo acomodar la pluralidad de concepciones políticas y doctrinales existentes, lo que Rawls llama "el hecho del pluralismo",

\footnotetext{
21 Appiah (2007, p. 211).

22 Mill (1869, p. 140).

23 A la luz del análisis de los variados códigos penales puede deducirse que el reconocimiento de la gravedad de estos crímenes trasciende las fronteras nacionales. Cf. Höffe (2007, p. 237).

24 Wellman (1996, pp. 211-237).

25 Maloberti (2009).
} 
dentro de un único esquema normativo. Partimos de que las personas son diferentes en temperamento, metas, dotes intelectuales y físicas y planes vitales y que, consiguientemente, no existe la posibilidad de crear un modelo óptimo para todos. Son numerosos los parámetros a considerar y muchas las decisiones que entran en juego en cada ordenamiento posible. La existencia de estas diferencias podría instar a renunciar a todo ideal cosmopolita. Nozick se plantea este mismo asunto y se pregunta cómo sería posible crear condiciones óptimas para personajes tan dispares como Wittgenstein, Elizabeth Taylor, Bertrand Russell y todo un largo elenco de personalidades variopintas y concluye que la idea de llegar a un modelo social óptimo para todos es sencillamente impracticable. 26

Puede que así sea si se persigue un modelo óptimo, pero no tanto si lo que se persigue es emplazar los pilares que permitan la convivencia pacífica, abandonando definitivamente la posibilidad de diseños ideales perfeccionistas o comunitaristas que nos llevarían "necesariamente arrastrados hacia los rápidos mortales de las utopías absolutas". 27

También es necesario conjurar el riesgo del etnocentrismo con la imposición universal de los propios valores, ideas y conceptos. Existe la tentación de pensar que el mayor despliegue de la razón tecnológica del mundo industrializado puede ser interpretado como muestra de superioridad general de razonamiento. A nuestro juicio, el despliegue técnico es la consecuencia de haber desarrollado un marco cultural que hace posible que seres humanos perfectamente comunes hayan refinado sus ideas gracias a complejas instituciones de investigación, reflexión y análisis. Las culturas científicas, muy superiores en cuanto a la comprensión de los hechos y la manipulación y transformación de la realidad, no están en posición de superioridad con respecto a otro tipo de valores. Es presuntuoso pensar que la superioridad en un campo implica automáticamente primacía en todos los demás.

Descartando modelos maximalistas, la alternativa sería la de llegar a un lenguaje compartido con el que se pudieran establecer los límites irrenunciables de la diversidad cultural y se abriera el resto del espacio a las diferentes formas de ser. ${ }^{28}$

Esto no podría hacerse sin el reconocimiento público de unos principios de justicia que todos acepten y sepan que los demás también aceptan. Esos principios compartidos habrían de reflejar los valores de consenso, es decir, los puntos de encuentro de las diferentes sensibilidades sociales, al menos en lo tocante a las bases para cooperar y dirimir los conflictos que surjan. Si no existe consenso sobre

\footnotetext{
26 Nozick (1999, pp. 310-311).

27 Castells (2001, p. 34).

$28 C f$. Fernández (2003). En este sentido, es también interesante señalar proyectos como el liderado por Hans Küng de desarrollar un ensayo de Ética Mundial a través de un Parlamento de las Religiones del Mundo, celebrado en Chicago en 1993, para llegar a enunciar máximas universalizables que se encuentran en las religiones y sistemas éticos del mundo.
} 
este marco básico, que incluye un esquema de responsabilidades mutuas, no es posible edificar una entidad global perdurable. La posibilidad de formalizar un acuerdo global pasa por tanto por intentar coincidir en torno a principios de la justicia aplicables universalmente, al margen del lugar o las particularidades de sus habitantes.

En segundo lugar, es imprescindible que tales valores se vean materializados en el funcionamiento de las principales instituciones políticas y sociales de un modo tal que todos puedan comprobarlo fehacientemente. La estructura resultante, en la medida en que se apoya en principios comunes favorece la cooperación. Nos parece pertinente matizar que la cooperación social no puede confundirse con la mera coordinación. La coordinación social de una actividad puede ser llevada a cabo por alguna autoridad, mientras que el concepto de cooperación tiene otras implicaciones: ha de estar regulada mediante reglas y procedimientos públicamente admitidos y plenamente aceptados por los cooperantes, que se convierten en los actores y guionistas de sus conductas cooperativas. El concepto de equidad va ligado al de cooperación, puesto que para que todos lleguen a cooperar es necesario que acepten las normas que organizan la cooperación, para lo cual, estas deben afectar por igual a todos y asegurar que el reparto de obligaciones se hace conforme a criterios que todos pueden llegar a aceptar voluntariamente. Lo más que podemos decir es que aunque la equidad no es la garantía del cumplimiento de las normas, su ausencia sí avala el incumplimiento.

Trasladados a una sociedad abierta y global, estas dos condiciones se traducen en la búsqueda de valores mínimos compartidos y su traslación en una estructura institucional global.

De modo que el principal problema a solventar de cara a lograr la estabilidad de una entidad política compartida sería hallar un consenso de valores que pudieran defenderse como los fundamentales. ${ }^{29}$ El problema es que no se trata de principios obvios. Es ingenuo pensar que el diálogo puede llevar a alcanzar acuerdos con facilidad. Los mismos valores admiten multitud de matices y pueden plasmarse de muy diversos modos, por no hablar de la dificultad que entraña jerarquizarlos. La idea de justicia no es un concepto exento de polémicas, existen diversas concepciones de lo justo y lo injusto. El conflicto entre valores no es de fácil resolución porque llega un punto en que es necesario sacrificar unos valores en favor de otros.

Siendo así, una buena manera de dirimir los conflictos de este tipo es permitir que exista suficiente publicidad y libertad. La fórmula trascendental del derecho público de Kant postula que son injustas todas las acciones que se refieren al derecho de otros hombres cuyos principios no soportan ser publicados. ${ }^{30}$ Una vez publi-

29 Ibid., p. 96 y Blanco (2005, p. 387).

${ }^{30}$ Kant (2003, pp. 61-62). 
citadas, las ideas contrapuestas deben poder competir ordenadamente entre ellas durante un buen periodo de tiempo. 31

En virtud del principio jurídico negativo de Kant, no serían aceptables todas aquellas que no soportaran la claridad de la publicidad y la embestida de las críticas. Por ello, una de las medidas esenciales de llegar a valores compartidos, máxime cuando se apunta a un entorno global, es la existencia de foros de debate público simétricos apoyados por un amplio abanico de medios de comunicación con distintas sensibilidades que garanticen una pluralidad de puntos de vista, presentados de una manera profesional y competente. En estos foros es donde se puede defender o discutir la vigencia de los valores sobre los que edificar la sociedad política. Las distintas doctrinas existentes intentarán presentar su esquema general y sus jerarquías, pero esas cuestiones no son determinantes. De lo que se trata más bien es de que expliciten públicamente en términos comprensibles para los demás cuáles son las razones por las que estiman que dichos valores son deseables y por qué serían indeseables los opuestos. Todo valor que pretenda convertirse en fundacional ha de basarse en razones que todos puedan compartir, o expresado en términos negativos, ha de ser un valor ante el que nadie pueda plantear objeciones razonables.

Nos estamos refiriendo en todo momento al plano público pues entendemos que los debates doctrinales no son competencia del Estado. En la esfera pública es donde es necesario establecer valores públicos sobre los que sí es exigible rendir cuentas, es decir, ofrecer justificaciones y argumentos públicamente comprensibles si se presentan como aspirantes a regular el plano público.

Llegados a este punto conviene introducir un ápice de escepticismo. No queremos soslayar las dificultades de implementación de estas propuestas, tanto por el proyecto cosmopolita en sí, como por los problemas técnicos aparejados (publicidad, espacios de diálogo, simetría de los interlocutores, etc.) o por la evidente dificultad de unificar criterios y establecer consensos universales. En todo caso, y sin soslayar estas trabas, entendemos que una propuesta teórico-normativa genera necesariamente una tensión con el plano de los hechos empíricos en el que habría de materializarse. Teniendo esto en cuenta, pasamos a continuación a presentar estrategias y razones que invitan a considerar que la posibilidad de un acuerdo global no es del todo implausible.

En primer lugar, nos parece relevante destacar que acaso una de las posibilidades más fructíferas de llegar a acuerdos entre personas y pueblos de diferentes tradiciones sea concentrarse en debatir acerca de qué se considera correcto o incorrecto con independencia de las razones, motivos, intuiciones o doctrinas por las que se llega a tales conclusiones. Es posible que unos fundamenten sus valores en su religión o filosofía, en los códigos de sus mayores, en sus experiencias personales o en

31 Rawls (1996, p. 262). 
su particular ideología. El universo de justificaciones puede ser tan variado como las personas. Pero conviene recordar que el objetivo no es la concordancia teóricoargumentativa sino un hecho pragmático: que las diferentes sensibilidades lleguen a un acuerdo cuyos requerimientos no choquen frontalmente con sus intereses esenciales o los valores básicos de los pactantes. 32

Así concebidos, los valores serían un instrumento cuya validez se mediría en la práctica. Entendemos que los valores son patrones de actuación reconocibles, una suerte de lenguaje que sirve para coordinar las vidas de quienes comparten espacio y tiempo. Se apela a ellos para dialogar y son uno de los más poderosos recursos que tenemos para cooperar con otros y evitar conflictos. Su función es por tanto eminentemente práctica: nos ayudan a actuar juntos, uniformizan reacciones y sentimientos ante los hechos del mundo.

Resulta apropiada la analogía con el lenguaje; son una construcción colectiva, el vocabulario de los valores se construye socialmente. Tan ilógico es un lenguaje individual como valores al margen del consenso social. Los valores son herramientas que dependen de consensos sociales que unos individuos comparten con otros. Así como en los lenguajes hay construcciones incorrectas por asintácticas, determinadas acciones pueden ser interpretadas, desde los códigos de valores, como incorrectas. Si partimos de que los valores son construcciones con un fin práctico y no modelos teóricos, parece posible llegar a encontrar algunos puntos de anclaje entre diferentes axiologías por medio de nuevas construcciones colectivas.

En segundo lugar, tomar en serio las diferencias de modos de vida y aspiraciones en el planeta nos lleva ciertamente a reducir las pretensiones del acuerdo político global. Es utópico, en el peor sentido del término, pretender llegar al consenso en todas las cuestiones, tal vez no sea ni siquiera deseable. Pero ello no implica abandonar el ideal de la extensión global de las condiciones de justicia.

Afirmar el universalismo de determinados principios no tiene por qué suponer la asimilación y anulación de la multiplicidad de modos de entender y conducirse por el mundo. La meta es diseñar una estructura compartida capaz de universalizar la posibilidad de que todos puedan vivir sus diferencias, conforme a su propio concepto del bien y su particular ética de máximos. ${ }^{33}$ Un marco jurídico global no exigiría conversiones ni renuncias inaceptables de las éticas de máximos y podría abrir el mayor margen posible a las idiosincrasias siempre y cuando no se violara un núcleo mínimo de valores políticos fundamentales.

El edificio de la comunidad política debe hacerse descansar en cimientos que para ser resistentes han de ser poco numerosos. Ambos conceptos van de la mano, pues sólo en torno a un limitado número de asuntos es posible alcanzar un compromiso global. La cooperación social estable se fundamenta en los valores más mode-

32 Rawls (1996, pp. 165-166).

33 Espinosa (2009). 
rados y menos controvertidos. El acuerdo demanda el abandono de las grandes pretensiones; no es razonable pretender regular y uniformizar todas las actividades humanas. Hay determinados valores que pueden ser universalizados, mientras que otros son necesariamente locales y no tienen por qué ser compartidos por el resto.

Por tanto, constreñir el acuerdo parece que es la única opción. No existen respuestas aceptables para todos en todas las cuestiones, tan solo puede hallarse una respuesta compartida y satisfactoria a un pequeño subconjunto de ellas. Lo esencial es identificar cuáles son estas sobre las que sí cabe el acuerdo y abordar de entre ellas las más urgentes con el fin de que las instituciones, basadas en estos principios compartidos, empiecen a ser efectivas. ${ }^{34}$ A nuestro juicio, tales valores mínimos deberían orbitar en torno a los derechos y libertades básicos e inviolables de los ciudadanos por un lado, los distintos poderes políticos y reglas democráticas por otro y las bases de los deberes samaritanos de asistencia mutua. Es básico reconocer áreas de inmunidad a los individuos, su derecho a tomar parte en el gobierno democrático de la comunidad y el marco común de solidaridad.

Por otro lado, es necesario reconocer que el hipotético consenso alcanzado sería de naturaleza inestable. Los valores están en conflicto permanente y las colisiones sociopolíticas en torno a ellos son insolubles. La historia nos da suficiente ejemplos de que no hay posibilidad de llegar a articular una constelación de valores armónicamente jerarquizados de una vez y para siempre. Tomar conciencia de la posibilidad continua del conflicto es el primer paso para empezar a adoptar medidas porque sólo se puede combatir lo que se conoce. No obstante, aunque los conflictos sean inevitables, los choques pueden amortiguarse y reconducirse dentro de los límites de la confrontación política. ${ }^{35}$

Algunas de las dificultades mencionadas pueden considerarse menores si entendemos que el consenso de mínimos no es una meta cerrada de una vez por todas. Por el contrario, es más factible entenderlo como algo abierto, susceptible de ser revisado a la luz de nuevos argumentos para adaptarse a los nuevos contextos.

Un motivo para el optimismo lo da el constatar que no partimos de cero a la hora de sentar las bases sobre las que podría construirse un consenso de valores mínimos. Puede hallarse un conjunto de ideas intuitivas fundamentales latentes en diversas culturas sobre las que edificar los cimientos éticos de una política global. ${ }^{36}$ Es posible encontrar valores generales que son compartidos por muchos hombres: acaso el principio más genérico sea tratar a los demás como uno mismo espera ser tratado, que, bajo distintas formulaciones, está presente en todas las civilizaciones. ${ }^{37}$ Confucio, Mahabharata, el Budismo, el Antiguo Testamento, el Corán y el

\footnotetext{
34 Rawls (1996, p. 188).

35 Berlin (1998, p. 153).

36 Rawls (1996, p. 207).

37 Höffe (2007, p. 153).
} 
Talmud judío lo presentan por la vía negativa, instando a no hacer a los demás lo que uno no desea para sí "Lo que es odioso para ti, no se lo hagas a tu prójimo. Esto es la totalidad del Torá; todo lo demás es comentario. Ve ahora y apréndelo". ${ }^{38}$ Los escritos zoroastrianos y el Nuevo Testamento toman la fórmula afirmativa "todo lo que queráis que hagan los hombres con vosotros, hacedlo vosotros con ellos"39 o "[y] lo que queréis que hagan a vosotros los hombres, eso mismo haced vosotros a ellos". 40 Puede decirse que la regla de oro, como se conoce esta máxima, está presente en las principales corrientes religiosas y filosóficas del mundo. El ideal que subyace es el de la igualdad y la reciprocidad, que nace de la creencia en la identidad moral de la persona. Todo ser humano tiene, por el hecho de serlo y al margen de fronteras territoriales y jurisdicciones políticas, derecho a las mismas libertades básicas como la de vivir su vida en libertad, criar sus hijos dignamente, no padecer hambre, violencia, injusticias u opresiones de ningún tipo y habitar con las condiciones mínimas de justicia social, especialmente en las necesidades humanas elementales, sin las cuales se hace difícil hablar de la dignidad del hombre.

Este principio general es parte de la plataforma igualitarista que encontramos en la mayoría de las teorías de filosofía política. ${ }^{41}$ Puede que muchos valores generales como la libertad y la igualdad conduzcan a anfibologías o contradicciones y no sean tan fundantes como el derecho a la igualdad de derechos. A diferencia de estos, el derecho a la igualdad de derechos no admite interpretaciones ni puede suscitar debates hermenéuticos. Es un principio rotundo y conciso. ${ }^{42}$ Esto es justo lo que Rawls establecía desde la primera formulación de su primer y más importante principio: que cada persona tenga el mismo derecho que cualquier otra al mayor esquema posible de libertades iguales para todos. 43

A pesar de la diversidad doctrinal razonable es viable alcanzar un consenso político en cuyo centro estén las nociones de igualdad y reciprocidad. Estas nociones pueden llegar a convenir a todas las partes por igual, porque apuntan al interés de todos.

Esta base compartida nos anima a considerar que las culturas no están tan alejadas en lo esencial: los valores fundamentales que inspiran los códigos de conducta y los principios básicos de la justicia, dejando al margen lo ritual o accesorio, no son tan distantes. Los valores no son idiomas intraducibles, hay grandes principios que actúan como "normas gramaticales universales" que pueden encontrarse en el lenguaje axiológico de todas la culturas humanas. ${ }^{44}$

\footnotetext{
38 Talmud, Shabbat 31 a.

39 Mt. VII, 12.

40 Lc. VI, 31.

41 Kymlicka (1995, passim).

42 Bermudo (2011).

43 Rawls (1999, p. 53).

44 Espinosa (2009).
} 
Los mitos, historias, narraciones, folklore, ópera, teatro, novelas, cuentos, ficción o no ficción, etnografía, pintura y fábulas muestran que, a pesar de que existen distintos contextos interpretativos, valores como la libertad, igualdad, autonomía o dignidad son universales. Ninguna cultura valora la cobardía o la mentira.

Este hecho no niega que exista una auténtica polifonía de valores en las sociedades complejas y plurales de hoy, pero sí permite albergar esperanzas de acercamientos entre ellas que permitan la mutua comprensión. Por comprender entendemos llegar a imaginar cómo piensan, sienten y viven los otros. Comprender no es sinónimo de compartir, pueden darse simultáneamente el rechazo y la simpatía, como nos demuestra continuamente la literatura. De modo que la incomprensión hacia una jerarquía de valores ajena no es incompatible con el respeto.

Si ha sido posible que en las sociedades nacionales, en cuyo seno conviven muy diferentes sensibilidades, se llegara a acuerdos axiológicos mínimos, parece que la empresa no habría de ser inabordable en mayores extensiones. Puede que en muchos casos, evidentemente, no haya concordancia, pero al menos es posible llegar a comprender las razones por las que se da el desacuerdo haciéndolo aceptable y conviviendo pacíficamente con él. Muchos de los principios de otras culturas pueden llegar a hacerse inteligibles si se hace un esfuerzo y si se cuenta con los instrumentos necesarios para realizar un acercamiento.

Además, hemos de sumar otro factor que hacen más factible la posibilidad de llegar a un pacto de estas características. El hecho de que no haya doctrinas con respuestas y valores para todos los aspectos vitales facilita el consenso, que se edifica sobre elementos compartidos y sobre espacios no ocupados por las doctrinas comprehensivas. Gracias al carácter incompleto de las doctrinas, creencias y confesiones es posible dialogar y acordar un punto de equilibrio estable en torno a una base moral adoctrinal, no relacionada directa y necesariamente con ninguna creencia, si bien compatible con todas ellas.

Pensamos que si reducimos los objetivos a la coexistencia pacífica en condiciones aceptables para todos, no se trata de un imposible; seguimos a Appiah cuando sostiene que los vocabularios axiológicos de todas las culturas se intercalan lo suficiente como para iniciar una conversación. ${ }^{45}$ Este punto de partida nos permite confiar en que no existen impedimentos de peso para rechazar la idea de que se pueda llegar a un mínimo común denominador moral, de aplicación universal, que, sin rechazar las diferencias de un mundo plural y sin pretender llegar a propugnar una cultura, ideología o religión como la mejor posible, sí permita hablar de acciones correctas o incorrectas, con independencia de quién las realice o dónde se realicen.

Creemos que no es ilógico sostener que es posible hallar un núcleo de valores sobre los que, sin miedo a pecar de etnocentrismo, se pueda edificar una sociedad

45 Appiah (2007, p. 91). 
política compuesta por individuos iguales con independencia de cuál sea su origen, raza o doctrinas generales. Lo que defendemos con estas consideraciones, y con las debidas precauciones, es que es posible ser moderadamente optimista acerca del acuerdo global de mínimos en torno a la igualdad de derechos a la luz de lo mucho que se ha avanzado en este terreno. Las diferencias culturales, lingüísticas o doctrinales no habrían de ser un obstáculo insalvable para llegar a un marco supraestatal en el que los conflictos pudieran resolverse mediante instrumentos políticos basados en consensos mínimos.

Con estas consideraciones en mente es posible concluir que es cierto que no está garantizado que de un debate político, ya sea con vecinos o con extraños, se vaya a llegar a un acuerdo definitivo. ${ }^{46} \mathrm{El}$ debate está destinado a no agotarse nunca y los acuerdos y soluciones de compromiso nunca serán absolutos porque no hay argumento con capacidad de persuadir a todos.

Sin embargo, lo importante es que las vías de comunicación permanezcan abiertas. El propio hecho de debatir ya implica partir de un valor mínimo compartido; quien acepta el diálogo se coloca ante un interlocutor al que respeta. La postura del que debate supone el reconocimiento implícito de que es necesario justificar lo que se piensa o hace ante otro, que es considerado legítimo acreedor de explicaciones. En ese sentido, debatir es incluir al otro. Por eso nunca se recalcará suficientemente la importancia que tiene mantener abierto el diálogo y la deliberación entre los miembros de la comunidad internacional en torno a los valores sobre los que erigir la convivencia global. Esta búsqueda de valores de consenso ya pone la bases para frenar los conflictos. ${ }^{47}$ Buscar una salida dialogada, crea hábitos de diálogo y cooperación, satisface la recíproca necesidad de acostumbrarse a la presencia del otro $\mathrm{y}$ es el inicio de un modus vivendi. Se trata de un proceso que requiere paciencia y tiempo, pero los primeros pasos ya son parte del camino.

\section{Referencias bibliográficas}

Appiah, K. A. (2007): Cosmopolitismo. La ética en un mundo de extraños. Buenos Aires-Madrid, Katz.

Bauman, Z. (1999): La globalización. Consecuencias humanas. Buenos Aires, FCE.

Bauman, Z. (2009): Tiempos líquidos. Vivir en una época de incertidumbre. (2 ${ }^{\mathrm{a}}$ edición ed.). (C. Corral Santos, Trad.) Barcelona, Tusquets Editores.

BECK, U. (1998): ¿Qué es la globalización? Falacias del globalismo, respuestas a la globalización. Barcelona, Paidós.

46 Ibid., pp. 74-76.

47 Appiah (2007, p. 115). 
BeCK, U. (2008): Las raíces cosmopolitas de la democracia: el caso de la Unión Europea. Sistema (206), 3-20.

Berlin, I. (2010): The Pursuit of the Ideal. En P. Kelly (ed.), British Political Theory in the Twentieth Century. Oxford: Wiley-Blackwell, pp. 143-155.

Bermudo Ávila, J. M. (2011): La libertad neorrepublicana. En J. M. Bermudo Ávila, \& (coord.), Figuras de la emancipación. Barcelona, Horsori.

BIEHL, J. (1998): The Politics of Social Ecology. Libertarian Municipalism. Québec, Canada, Black Rose Books.

Blanco Fernández, D. (2005): «Patriotismo.» En Democracia y virtudes cívicas, editado por Pedro Cerezo Galán. Madrid, Biblioteca Nueva.

Bodin, J. (1985): Los seis libros de la República. Madrid, Editorial Tecnos.

Castells, M. (2001): La era de la información. Fin de milenio. Vol. 3 . 3 vols. Madrid, Alianza Editorial.

Del Águila, R., \& Vallespín, F. (2006): Ideologías políticas y futuro. En J. A. Mellón, ideologías y movimientos políticos contemporáneos. Madrid, Tecnos.

ESPINOSA ANTÓN, F. J. (2009): «¿Universalismo moral de la Ilustración versus multiculturalismo en las sociedades del siglo XXI?» Logos. Anales del Seminario de Metafísica (Servicio de Publicaciones de la Universidad Complutense de Madrid), pp. 219-241.

FERNÁNDEZ DEL RIESGO, M. (2003): «Globalización, interculturalidad, religión y democracia.» Ilu. Revista de ciencias de las religiones, $\mathrm{n}^{\circ}$ 8, pp. 5-27.

Giddens, A. (1987): Social Theory and Modern Sociology. Oxford, Polity Press.

Gilpin, R. (1981): War and Change in World Politics, Cambridge, Cambridge University Press.

Habermas, J., Held, D., y Kymlicka, W. (2005): «Declaración de Granada sobre la globalización.» El País, 6 de junio, p. 14.

Hardt, M., y Negri, A. (2002): Imperio. Barcelona, Ediciones Paidós Ibérica.

HeLd, D. (2005): Un pacto global. La alternativa socialdemócrata al consenso de Washington. Madrid, Santillana Ediciones Generales S.L.

Held, D. (2007): Modelos de democracia ( $3^{\mathrm{a}}$ ed.). (M. Hernández, Trad.) Madrid, Alianza Editorial.

Hobsbawm, E. (1995): Historia del siglo XX. Barcelona, Crítica.

HöfFE, O. (2007): Ciudadano económico, ciudadano del Estado, ciudadano del mundo, Buenos Aires, Katz Editores.

Kant, I. (2003): De lo bello y lo sublime. La paz perpetua. Madrid, Editorial Espasa.

Maloberti, N. (2009): Libertarianism and the Possibility of the Legitimate State. Libertarian Papers 1, 5. Online: www.libertarianpapers.org. , 1 (5), 1-12.

MiLl, J. S. (1869): On Liberty (4 ed.). London, Longmans, Green, Reader and Dyer. MiLl, J. S. (1997): El utilitarismo. Madrid, Alianza Editorial. 
NozICK, R. (1999): Anarchy, State, and Utopia. Oxford: Blackwell Publishers Ltd. RaWls, J. (1996): El liberalismo político. Barcelona, Crítica S.L.

Rawls, J. (1999): Theory of Justice Rev. Ed. Cambridge, Mass., Harvard University Press.

RAWLS, J. (2001): El derecho de gentes y "Una revisión de la idea de razón pública”. Traducido por Hernando Valencia Villa. Barcelona, Paidós.

SASSEN, S. (2007): Territorio, autoridad y derechos. De los ensamblajes medievales a los ensamblajes globales. Traducido por María Victoria Rodil. Madrid, Katz Editores.

Singer, P. (1972): "Famine, Affluence and Morality," Philosophy and Public Affairs 1.

Toulmin, S. (2001): Cosmópolis. El trasfondo de la modernidad. Barcelona, Ediciones Península.

Unger, P. (1996): Living High and Letting Die: Our Illusion of Innocence, New York, Oxford University Press.

Wellman, C. H. (1996): Liberalism, Samaritanism, and Political Legitimacy. Philosophy and Public Affairs , 25 (3), 211-237.

Juan Antonio Fernández Manzano

Dpto. Filosofía del Derecho, Moral y Política II (Ética y Sociología)

Facultad de Filosofía

Universidad Complutense de Madrid

jafmanzano@filos.ucm.es 